\title{
Editorial
}

\section{Do you Recommend an Interdisciplinary Field to your Graduate Student?}

\author{
Ali-Akbar Haghdoost ${ }^{1}$, Arash Shahravan ${ }^{2, *}$ \\ ${ }^{1}$ The Research Center for Modeling in Health, Institute for Futures Studies in Health, Kerman University of Medical Sciences, Kerman, Iran \\ ${ }^{2}$ Kerman Oral and Dental Diseases Research Center, Kerman University of Medical Sciences, Kerman, Iran
}

Received: 1 June 2013, Accepted: 12 June 2013, ePublished: 12 June 2013

"I would like to work on a very advanced, novel, and appealing topic; do you recommend working on the cost analysis of nano-surgeries?" A Masters student asked her supervisor. What would you suggest if that supervisor were you?

Why do students and even senior researchers look for a very advanced research topic? Is it the one and only way to work on the edge of science? What is the best way to express something, which is innovative and original?

Looking back over the history of science, medicine in particular, one can see a surge in the number of scientific fields in the recent centuries; i.e. many new branches have been observed to mount up. Nowadays, a clinical hematologist visits only patients with blood disorders; however, he has studied internal medicine in every respect before concentrating on a specific specialty. The gap among adjacent disciplines is widening to an extent that makes it hard to believe that only a few decades ago, a surgeon would operate all body parts. This branching phenomenon is visible in basic sciences as well. Many molecular fields are spanking new and the speed of their disjointing is even more than those of clinical fields.

This diversion in scientific fields opens new horizons for researchers. Researchers usually target advanced topics in the newly born branches to find a novel topic for their research; topics that are very specific and expensive and require high -tech facilities (1).

Although still most of the new knowledge is developing as the results of diversion of scientific fields, it is not the only way to express something innovative. Similarly, conversion of scientific fields plays an important role in bringing about bright innovations through merging different disciplines. This concept is so fundamental that it has been regarded as the third revolution in the history of sciences $(2,3)$.

visiting an exhibition about the concept of birth and death at microscopic level a few years ago, it was very interesting to see how art, life, and cellular biology are combined. The best selected picture in this exhibition showed how a colony of microorganisms flourished and expanded its territories, illustrating the reality of life and existence.

Form this perspective, many new research fields are recently introduced to researchers. Combination of geography and public health, literature and medicine, social sciences and epidemiology, journalism and public health management, and law and medical economy are examples of conversion in different scientific fields (1).

A shift in one's research field can bring new ideas and viewpoints to that new field. Organizations have to pave the way to make sure different experts with distinct backgrounds have enough contacts to fully use their capacities in addressing new research questions $(4,5)$.

The concept of conversion may introduce significant changes in the structure of universities as well as health systems. To put it another way, we may need experts from different disciplines at the universities of medical sciences. Accordingly, the title of our departments may change in the near future $(2,3)$. For instance, the department of epidemiology is not an appropriate title if we have a mixture of experts from different fields of epidemiology, biostatistics, health management, health economy, sociology, biology, mathematics, history, geography, preventive medicine, and so on.

This new interdisciplinary approach is a real need now. For example, how we can address the HIV pandemic comprehensively while diverse factors contribute to its development. Medical aspects such as care and treatment, vaccine development, virology, epidemiology and immunology constitute only one group of factors. This infection has social, economic, psychological, legal, and political aspects as well. As such, an isolated specialist team trained only in a unique discipline cannot explore and address this infection on its own. HIV was an example; for sure interdisciplinary approach to distinct health issue is essential today. Despite this need, the structure of our universities does not readily support and welcome research groups from different disciplines working together (1).

It is also the case for the title of journals. Nowadays, the number of very specialised journals is fast growing; those

*Corresponding author: Arash Shahravan; Email: arashahravan@gmail.com 
that publish extremely precise topics in very advanced subspecialties. At the same time, we have to think about interdisciplinary journals to publish the papers with a different approach. This is not a new concept and the number of inclusive journals is relatively high. However, most of these journals are somewhat old and were established a few decades ago (6). What we are proposing here is to publish a new generation of general journals to materialize this concept, and to support researchers for publishing the results of their interdisciplinary studies. A journal not only to publish papers from different disciplines, but also to accept cross-disciplinary papers which are the product of convergence in science.

The "International Journal of Health Policy and Management" (IJHPM) is an attempt to respond to this need in public health field and encourages researchers to work in multi-disciplinary teams. With no doubt, the journal would be successful only if it receives high-quality papers from multidisciplinary experts in different countries. Therefore, we cordially invite you to contribute if you believe that we are on the same page.

In addition, we are writing this paper to open a discussion about the concept of conversion in public health sciences, and its impact on the production of new knowledge in the future. We expect to receive your letters and papers in response to this call, and hope to have a series of fruitful debates to be published in the next issues of the IJHPM.

Ethical issues

Not applicable.

Competing interests

None.

Authors' contributions

$\mathrm{AH}$ initiated the idea. Both authors contributed to the drafting of the initial manuscript Both authors contributed to the editing and revising of the final manuscript.

\section{References}

1. Grigg L. Cross-disciplinary research. Australian Research Council 1999.

2. Sharp PA, Cooney CL, Kastner MA, Lees J, Sasisekharan R, Yaffe MB, et al. The third revolution: the convergence of the life sciences, physical sciences, and engineering. Massachusetts Institute of Technology 2011. 3. Sharp PA, Langer R. Promoting convergence in biomedical science. Science 2011; 333: 527.

4. Hindle T, Checkland P, Mumford M, Worthington D. Developing a methodology for multidisciplinary action research: a case study. Journal of the Operational Research Society 1995; 46: 453-64.

5. Nelson B. Interdisciplinary studies: seeking the right toolkit. Nature 2011; 476: 115-7.

6. Porter AL, Chubin DE. An indicator of cross-disciplinary research. Scientometrics 1985; 8: 161-76. 